\title{
Intersections
}

Canadian Journal of Music

Revue canadienne de musique

\section{Theodor Dumitrescu, Karl Kügle et Marnix van Berchum (dir.). 2013. Editing Early Music: Principles, Historiography, Future Directions. Turnhout : Brepols. 290 p. ISBN : 978-2-503-55151-7}

\section{Geneviève Barbara Bazinet}

Volume 35, numéro 2, 2015

URI : https://id.erudit.org/iderudit/1043823ar

DOI : https://doi.org/10.7202/1043823ar

Aller au sommaire du numéro

Éditeur(s)

Canadian University Music Society / Société de musique des universités canadiennes

ISSN

1911-0146 (imprimé)

1918-512X (numérique)

Découvrir la revue

Citer ce compte rendu

Bazinet, G. B. (2015). Compte rendu de [Theodor Dumitrescu, Karl Kügle et

Marnix van Berchum (dir.). 2013. Editing Early Music: Principles,

Historiography, Future Directions. Turnhout : Brepols. 290 p. ISBN :

978-2-503-55151-7]. Intersections, 35(2), 119-124.

https://doi.org/10.7202/1043823ar

Copyright @ Canadian University Music Society / Société de musique des universités canadiennes, 2018
Ce document est protégé par la loi sur le droit d'auteur. L'utilisation des services d'Érudit (y compris la reproduction) est assujettie à sa politique d'utilisation que vous pouvez consulter en ligne.

https://apropos.erudit.org/fr/usagers/politique-dutilisation/ 


\section{BOOK REVIEWS / RECENSIONS}

Theodor Dumitrescu, Karl Kügle et Marnix van Berchum (dir.). 2013. Editing Early Music: Principles, Historiography, Future Directions. Turnhout: Brepols. 290 p. ISBN : 978-2-503-55151-7.

Les éditions de musique ancienne («early music» en anglais) empruntent de nombreuses formes depuis plus de cent ans: les éditions critiques, les éditions exécutables ("performing editions»), les éditions de compositeur, les éditions qui reproduisent un seul manuscrit et les éditions d'anthologie qui rassemblent des pièces s'inscrivant dans un même genre. Les pratiques éditoriales de ces publications au fil des ans ont aussi été variées, s'appuyant sur la méthodologie de la critique textuelle littéraire comme la philologie lachmanienne ou l'approche de «manuscrit de base» de Joseph Bédier, ou en fournissant des transcriptions «diplomatiques» qui visent à transcrire et à moderniser le texte original. Les éditeurs modernes continuent de traiter du problème de pratiques éditoriales désuètes, non conventionnées, et ne sont toujours pas d'accord en ce qui a trait au format de l'édition et aux types de renseignements que le musicologue devrait fournir à ses lecteurs ${ }^{1}$.

Dirigé par Dumitrescu, Kügle et van Berchum, ce volume de la collection «Épitome musical» Early Music Editing: Principles, Techniques, and Future Directions, rassemble une sélection de communications de plusieurs musicologues de grande renommée qui ont été présentées lors d'un colloque éponyme à Utrecht en 2008. Cet ouvrage collectif est un ajout opportun à la discussion en cours sur la façon d'éditer la musique ancienne ${ }^{2}$. La pluralité des points de vue présentés dans le livre représente un large éventail d’opinions sur les pratiques éditoriales de la musique ancienne. Les auteurs décrivent les défis inhérents à certains répertoires et posés par plusieurs approches éditoriales, et proposent des solutions possibles pour quelques problèmes spécifiques, bien quaucun ne prétende avoir trouvé une approche universelle pour l'édition de cette musique.

1 David Fallows a récemment annoncé qu'il ne comprendrait pas les variantes des ligatures dans son édition Secular Polyphony 1380-1480 pour Musica Britannica. (Fallows 2013, p. 104-107).

2 Le thème de l'édition musicale a été le sujet de plusieurs colloques internationaux depuis 2008, tel que: "The Editing of Historical Music in the $21^{\text {st }}$ Century-Conference on Music Editing", Oslo, Norvège, 10-11 décembre 2010; les divers événements liés au Music Encoding Initiative (MEI), y compris la Music Encoding Conference, Florence 18-21, 2015; des ateliers lors de colloques annuels tels que le colloque annuel de l'AMS à Pittsburgh, 2013. De nouveaux projets en ligne, comme le «Du Chemin Lost Voices Project ", continuent à repousser et à élargir les frontières de l'édition de musique ancienne. 
En fait, l'affirmation que chaque répertoire, compositeur ou source nécessite une approche personnalisée pour l'édition se fait entendre tout au long du livre, tout comme l'espoir que les développements futurs dans la production d'édition critique «en ligne» offriront de nouvelles possibilités pour l'édition de la musique ancienne.

Louvrage collectif impressionnant de 290 pages aborde les nombreux défis éditoriaux de la musique ancienne, que ce soit l'Office Divin ou la musique pour clavier du XVII ${ }^{e}$ siècle. Il envisage également le potentiel des éditions critiques en ligne et examine la méthodologie et les pratiques éditoriales passées et actuelles.

L'introduction de Karl Kügle positionne d'emblée plusieurs sujets récurrents abordés tout au long du volume, plus particulièrement le débat concernant l'efficacité et la pertinence de la philologie dans l'édition de la musique ancienne ainsi que le concept de la «performativité» de la musique et son aire distinctive d'un art d'interprétation qui dépasse la notation écrite.

Les dix chapitres sont organisés en deux sections. La première section, plus développée, comprend les chapitres 1 à 7 qui traitent de répertoires précis et de pratiques de rédaction. La seconde section, qui inclut les chapitres 8 à 10, propose une analyse des genres d'édition depuis les premières technologies d'imprimerie jusqu'aux éditions numériques. Le dernier chapitre (conclusion de Margaret Bent) revient sur plusieurs de ces thèmes, et se penche plus particulièrement sur les principes d'éditions. Dans le cadre de cette recension, il ne sera pas possible de décrire en détail chacune des contributions, mais nous croyons utile d'en présenter les principaux éléments.

L'article intitulé «Do Classical Principles Work?» de Leofranc HolfordStrevens entame la discussion sur l'utilisation des méthodes philologiques dans l'édition de la musique ancienne. Holford-Strevens soutient que, pour la polyphonie de la Renaissance, la méthode stemmatique ${ }^{3}$ peut être très utile pour l'éditeur moderne en quête du texte original de l'auteur, le «Urtext ». Holford-Strevens maintient que les révisions et les corrections des copistes sont toutes des points d'intérêt, en particulier par rapport au «Urtext», et il prône l'incorporation des principes philologique dans le processus éditorial.

Dans son article «Editing the Divine Office», James Grier se penche sur les défis auxquels les éditeurs de ce répertoire doivent faire face, en posant la question suivante: "When one edits an Office, what, precisely is one editing? » (p. 32). Grier traite de deux de ses propres éditions, la première est une série d'offices compilés par Adémar de Chabannes et la seconde est une reconstruction de l'Office de la Trinité à Saint-Marital au XIe siècle. Les deux éditions ont nécessité des approches éditoriales différentes: l'une s'appuie sur la méthode stemmatique et crée un texte de compilation tandis que l'autre utilise l'approche de Bédier, appelée «manuscrit de base». Sur la base de son expérience, Grier

3 La méthode stemmatique, approche fondamentale de la critique textuelle, se penche sur l'étude des variantes des éditions existantes afin d'arriver à la reconstruction de l'histoire du texte. Le musicologue classe les éditions existantes par famille et crée un arbre généalogique (stemma codicum) selon les points communs des variantes. Il arrive finalement à un seul «archétype» duquel proviennent toutes les éditions. 
plaide pour des pratiques éditoriales transparentes, des décisions éditoriales explicitement disposées pour le lecteur et pour que ces décisions soient déterminées par le type d'édition et assez flexibles pour répondre aux exigences et aux besoins des lecteurs issus des communautés artistique et universitaire.

La contribution de Jason Stoessel, intitulée "Scribes at Work, Scribes at Play: Challenges for Editors of the ars subtilior», examine le rôle du copiste dans la transmission de sources d'ars subtilior. Stoessel favorise une approche de l'édition qui peut prendre en compte le contexte culturel et les pratiques musicales. Puisque le répertoire de l'ars subtilior n'était pas «stable», la philologie et l'approche "manuscrit de base» sont, d'après Stoessel, inappropriées et laissent de côté les "plausibles readings» (p. 58). Stoessel démontre que ces sources peuvent fournir des renseignements de valeur sur les activités des copistes et sur l'évolution de la compréhension et de l'utilisation de la notation musicale au XIV $\mathrm{V}^{\mathrm{e}}$ siècle. Il recommande la création d'éditions critiques en ligne qui pourraient inclure toutes les leçons et permettre aux lecteurs de former leurs propres leçons.

Andrea Lindmayr-Brandl souligne l'importance d'une des premières éditions critiques de musique ancienne, le Codex de Trente, publiée entre 1900 et 1933 dans le cadre de la série Denkmäler der Tonkunst in Österreich, éditée par Guido Adler et Rudolf von Ficker. Lindmayr-Brandl analyse les pratiques éditoriales utilisées par Adler et von Ficker tout au long de leur édition. L’article se termine avec une reproduction de la préface de l'édition de 1933 de von Ficker qui fut récemment découverte et qui traite de son utilisation d'une notation particulière dans son édition 4 .

Le chapitre de Thomas Schmidt-Beste aborde le sujet complexe du placement du texte, une caractéristique de la musique ancienne qui a souvent été rejetée comme étant trop transitoire et changeante pour être incluse dans la rédaction d'éditions critiques ${ }^{5}$. L'enquête de Schmidt-Beste sur les pratiques éditoriales en ce qui concerne le placement du texte dans les éditions critiques souligne le fait que de nombreux ouvrages mettent peu d'emphase sur le catalogage attentif ou conséquent de variantes textuelles dans leurs appareils critiques tandis que les éditions qui essaient de reconstruire le placement du texte ne justifient généralement pas la méthode choisie. Bien que les éditions en ligne peuvent offrir de nombreuses solutions pour l'éditeur (telles qu'une édition synoptique avec des liens vers la version numérisée des fac-similés), Schmidt-Beste conclut que c'est la responsabilité de l'éditeur de fournir une solution éclairée du placement du texte dans son édition et qu'une considération égale doit être donnée aux mots ainsi qu'aux notes.

David J. Smith, dans «Editing Early Keyboard Music: The Role of the Scribe in the Transmision of Music by Peter Philips », discute des sources de la musique de Peter Philips et du rôle important du copiste dans la transmission de ce répertoire. Smith aborde également la question de la philologie concluant

4 Les commentaires de Lindmayr-Brandl au sujet de la préface sont disponibles dans : Lindmayr-Brandl 2012, p.103-140.

5 Voir Meconi 1992, p. 284-291. 
qu'un archétype reconstitué peut être aussi valable que l'approche «manuscrit de base» étant donné la nature fluide de ce répertoire.

Ouvrant la seconde section de l'ouvrage sur les genres d'édition, la contribution de Christine Jeanneret s'interroge sur l'impact que les diverses technologies utilisées pour reproduire la musique écrite (sous forme de manuscrits, d'impression et de gravure) ont eu sur la manière dont la musique a été notée. Jeanneret souligne l'importance des choix de technologie représentative et ses conventions, et que celles-ci, ainsi que les conventions historiques, géographiques et de notation, doivent être respectés, surtout pour les genres musicaux comme l'opéra ancien.

L'article d'Alexander Silbiger, "The Promises and Pitfalls of Online Scholarly Music Publishing» examine les différents types d'éditions en ligne et les premières épreuves dans la création et la publication des éditions en ligne. Lauteur présente les avantages de la publication en ligne, entre autres la capacité de mettre à jour rapidement l'édition et de fournir des hyperliens vers des documents supplémentaires, aussi bien que quelques inconvénients, tels que les défis de générer des revenus comparables aux éditions imprimées et l'aspect temporaire des éditions numériques, qui expliqueraient pourquoi les éditions en ligne n'ont pas encore supplanté leurs homologues imprimés. Il prend pour exemples différents types d'éditions disponibles en ligne (entre autres les démarches de la Web Library of Seventeenth-Century Music - WLSCM, et le projet Computerized Mensural Music Editing - CMME), et affirme que l'avenir des éditions critiques se situe dans le numérique ${ }^{6}$.

Theodor Dumitrescu se penche sur la problématique des méthodes philologiques et des pratiques d'édition antérieures. Il favorise l'approche théorique de la «new philology» en tant qu'alternative aux défis et aux lacunes des méthodes traditionnelles. Dumitrescu traite de l'implémentation de cette approche dans les éditions en ligne produite par Computerized Mensural Music Editing (CMME) en énumérant les diverses options disponibles par l'intermédiaire du site Web des éditions en ligne, y compris la capacité de trier les variantes de pièces de musique individuelles par genre (par exemple, afficher uniquement les variantes rythmiques ou les ligatures) ou encore de la possibilité d'afficher tous les relevés présentés sur la partition plutôt que dans un tableau d'abréviations, comme le fait l'appareil critique traditionnel.

Le dernier chapitre du volume, «Early Music Editing, Forty Years On: Principles, Techniques, and Future Directions» de Margaret Bent, était à l'origine le discours d'ouverture du colloque. Ici, Bent réexamine les préoccupations éditoriales telles que les valeurs de note, les accidents et les clefs, et elle discute de l'organisation des différents genres d'éditions de musique ancienne. Elle prône les éditions qui fournissent une grammaire musicale correcte pour ceux qui les utilisent. Selon Bent les éditions devraient être plus qu'une simple reproduction d'un texte: les éditeurs doivent corriger les erreurs dans les sources,

6 WLSCM a publié sept volumes depuis 2011 aussi bien qu'une nouvelle série (Monuments of Seventeenth-Century Music). "http://sscm-wlscm.org/». D'autre part, CMME semble avoir peu de nouvelles publications : la page «Latest news» date d'octobre 2012 et la dernière mise-à-jour de leurs projets actuels date de juin 2011. «http://cmme.org/about». 
indiquer tous les accidents et être au courant de la grammaire de la musique qu'ils présentent aux lecteurs (p. 267).

Le contenu de ce volume reflète plusieurs préoccupations courantes liées à l'édition de la musique ancienne telles que les activités du copiste, la pratique philologique et le potentiel des éditions critiques en ligne, mais une discussion des pratiques éditoriales des sources portant sur la théorie musicale est absente malgré son intérêt manifeste7. Il s'adresse principalement aux spécialistes qui retrouveront plusieurs points de vue importants et intéressants pour le développement de leur discipline. Cet ouvrage ne propose pas, cependant, de «bonnes pratiques» pour les étudiants ou novices, ou en vue d'améliorer les pratiques courantes, mais il présente plutôt les recommandations de «bons principes» de la part de chaque spécialiste par rapport à leur répertoire. Ainsi, on y retrouve plusieurs principes directeurs pour la révision de la musique ancienne, y compris des exemples concrets d'analyse pour certains répertoires particuliers. Parmi les principes récurrents recommandés par ces spécialistes se dégage le besoin d'une approche au cas par cas lors de la révision ainsi que la nécessité de comprendre la grammaire musicale et les conventions de la musique à réviser. Cet ouvrage nous offre un aperçu important des pratiques d'éditions de la musique ancienne ainsi que des informations pertinentes en ce qui a trait aux éditions existantes et à la discussion en cours sur les éditions numériques. L'intérêt de ce recueil sera noté non seulement par les musicologues, mais aussi par quiconque utilise les éditions de musique ancienne.

Lorganisation du volume pourrait être améliorée par le regroupement des chapitres par sujets communs, par exemple, le regroupement des contributions de Smith et de Stoessel au sujet des activités des copistes ${ }^{8}$. L'inclusion de soustitres dans la table des matières pour mettre en évidence les tendances générales explorées par les auteurs clarifierait le contenu du livre, compte tenu du fait que le volume ne comprend pas d'index. Il s'agit cependant d'une question de format qui ne diminue pas l'importance de l'information présentée ici ni la valeur du livre pour les professionnels et les étudiants et tous lecteurs qui s'intéressent au sujet de l'édition de la musique ancienne. Le volume est très certainement pertinent alors que nous continuons d'explorer les pratiques éditoriales de la musique ancienne et le potentiel d'éditions critiques en ligne.

Geneviève Barbara Bazinet

\section{RÉFÉRENCES}

Dumitrescu, Theodor, (dir.). «The Computerized Mensural Music Editing Project», http://cmme.org/about. . «Program». Early Music Editing: Principles, Techniques and Future Directions, http://earlymusicediting.cmme.org/program.html.

Fallows, David. 2014. Secular Polyphony 1380-1480. London : Stainer and Bell.

7 Une communication à ce sujet fut présentée lors du colloque en 2008. http://earlymusicediting.cmme.org/program.html.

8 L'organisation par sujet fut appliquée au programme du colloque en 2008. 
.2013. «A note on ligatures». Early Music, XLI : 104-107.

Freedman, Richard et Philippe Vendrix (dir.) «Lost Voices Project», http:// www.digitalduchemin.org/.

Lindmayr-Brandl, Andrea. 2012. "Rudolf von Fickers Einleitungstext zum sechsten Auswahlband der Trienter Codices in den DTÖ: Ein wiederaufgefundener Entwurf aus dem Nachlass». Rudolf von Ficker (1886-1954): Tagungsband zum Symposium anlässlich seines 125. Geburtstages und des 85-jährigen Bestehens des Innsbrucker Institutes für Musikwissenschaft, sous la dir. de Lukas Christensen, Kurt Drexel et Monika Fink, 103-140. Innsbruck: Innsbruck University Press.

Meconi, Honey. 1992. "Is Underlay Necessary?» Companion to Medieval and Renaissance Music, sous la dir. de Tess Knighton and David Fallows, 284291. Berkeley : University of California Press.

Tilley, Janette (ed.). «Monuments of Seventeenth-Century Music». Web Library of Seventeenth-Century Music, http://www.sscm-wlscm.org/.

\section{BIOGRAPHIE}

Geneviève Bazinet est professeure auxiliaire à l'École de Musique de l'Université d'Ottawa. Ses recherches portent sur la musique de la Renaissance, plus précisément sur l'imprimerie de Pierre Attaingnant et l'imprimerie musicale au XVI ${ }^{\mathrm{e}}$ siècle, ainsi que sur les livres d'Heures, sur la musique du cinéma, et sur la musique du cinéma muet au Canada. Elle a donné des conférences aux colloques annuels de l'American Musicological Society, Med-Ren, ainsi que la Société de musique des universités canadiennes. Bazinet participe au Groupe de recherche multidisciplinaire sur les livres d'Heures conservés au Québec ("Un Catalogue raisonné des livres d'Heures des XVe et XVI siècles conservés au Québec”) dirigé par Brenda Dunn-Lardeau (Université du Québec à Montréal), et au projet "Silent Film Music in Canada" dirigé par Paul Merkley (University of Ottawa). Ses recherches ont été publiées dans Renaissance et Réforme. Elle est récipiendaire de la Claude V. Palisca Fellowship in Musicology of the Renaissance Society of America (2017), bourse accordée pour sa recherche au sujet de la musique et les dévotions en France au XVI ${ }^{\mathrm{e}}$ siècle. 